\title{
Salvataje del pie diabético en el Perú: ¿un mito o realidad?
}

Diabetic foot salvage in Peru: a myth or reality?

\section{Señor Editor:}

Durante los últimos años, el aumento de la prevalencia de la Diabetes Mellitus (DM) (11\%, aprox. 3520000 personas) y de sus complicaciones vasculares crónicas, ha conllevado a la gran demanda de cirujanos vasculares y endovasculares en todo el Perú (1); estos ofrecen un manejo y tratamiento multidisciplinario de la insuficiencia arterial periferica (Salvataje del pie diabético) (2), el cual si no se trata oportunamente conlleva en sus estadios finales a un cuadro vascular de isquemia crónica que amenaza la extremidad (CLTI por sus siglas en inglés Chronic Limb-Threatening Ischemia) $(3,4)$.

Son muchas las instituciones hospitalarias en nuestro país que cuentan con un Servicio de Cirugía Vascular y Endovascular que vienen realizando un salvataje del pie diabético de primer nivel; es decir, la gran demanda de pacientes ha condicionado la implementación de técnicas endovasculares novedosas, profesionales altamente capacitados y amplia infraestructura hospitalaria permitiendo un óptimo desempeño multidisciplinario (Traumatología, Endocrinología, Medicina Física y Rehabilitación, etc...) que exije un salvataje del pie diabetico exitoso.

En la mayoría de los pacientes con DM admitidos para algun tipo de tratamiento endovascular, se evalúa clínicamente incluyendo el índice tobillo-brazo (ITB) y la ecografía Doppler vascular (EDV), permitiendo en un primer momento estratificar el riesgo de amputación del miembro inferior mediante el Score WIFI (Wound, Ischemia, and foot Infection) y posteriormente junto al estudio arteriográfico, clasificar la lesion vascular mediante el sistema GLASS (Global Limb Anatomic Staging System), para finalmente determinar si el paciente es o no tributario del tratamiento de revascularización abierta o endovascular $(3,5)$.

La revascularizacion endovascular junto a la curacion de heridas son consideradas en la actualidad, como la piedra angular de salvataje del pie diabético; debido a que se restituye adecuadamente el flujo sanguineo a zonas de la extremidad en donde antes no lo hubo, permitiendo contrarrestar los grados de isquemia crónica y propiciando la cicatrización exitosa de las heridas $(5,6)$. Para lograr todo este proceso terapéutico, el cirujano vascular y endovascular hace uso de una serie de herramientas que incluyen guías, catéteres, balones medicados, stens con droga y equipos de aterotomía, según la localización (Vaso Target y angiosoma), tipo y abordaje (anterógrado, retrógrado o contralateral) de la lesion a tratar (figura 1) $(7,8)$; por lo tanto, este proceso ha sido descrito como un verdadero desafío terapéutico debido a que se requiere una minuciosa planificación e innovacion de técnicas quirúrgicas teniendo en cuenta permanentemente la cartografía vascular a tratar $(9,10)$.

El salvataje de pie diabético, es una realidad palpable. A diario, nuestro servicio es afluente de muchos casos revascularizables procedentes de todo el Perú y en la actualidad estamos en pie de lucha contra dos grandes enemigos, la DM y la desinformacion que ha ocasionado en muchas oportunidades niveles de amputacion innecesarios en pacientes tributarios de terapia endovascular. Ante todo los mencionado, nace la

\footnotetext{
Departamento de Cirugía de Tórax y Cardiovascular, Hospital Nacional Edgardo Rebagliati Martins. Lima, Perú.

Área de Endocrinología, Clínica Delgado. Lima, Perú.

Médico Residente,

Médico Cirujano Cardiovascular y Endovascular.

Médico Endocrinólogo.

Maestro en Gerencia de Servicios de Salud.
} 


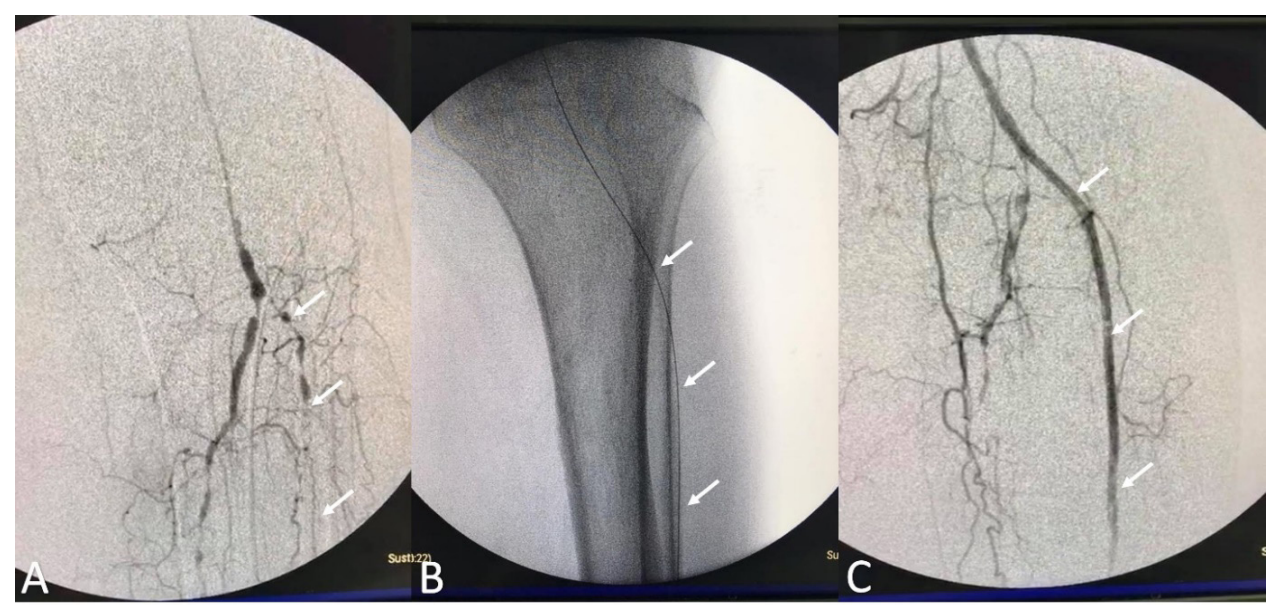

Figura 1. A: Obstrucción total de vasos infrageniculares donde se identifica a la arteria tibial anterior (ATA) como el vaso de prioridad a tratar (Vaso Target) (flechas). B: Se recanaliza ATA con el pasaje de una guía hidrofílica por acceso retrógrado (flechas). C: Angioplastía con balón de ATA desde acceso anterógrado luego de recapturar la guía (flechas).

necesidad de mayores investigaciones en la población de nuestro medio con la finalidad de estandarizar guías y protocolos de manejo multidisciplinario de pie diabético en las diferentes instituciones hospitalarias de nuestro país.

\section{W Samir Cubas ${ }^{1, a, d}$, Manolo Briceño-Alvarado ${ }^{1, b}$, Félix Tipacti-Rodríguez ${ }^{1, b}$, Helard Manrique-Hurtado ${ }^{2, c}$.}

\section{Correspondencia:}

Wildor Samir Cubas Llalle

Hospital Nacional Edgardo Rebagliati Martins, Servicio de Cirugía de Tórax y Cardiovascular

Av Edgardo Rebagliati 490, Jesús María, Lima, Perú.

Correo electrónico: wsamircubas@gmail.com

\section{REFERENCIAS BIBLIOGRÁFICAS}

1. Carrillo-Larco RM, Bernabé-Ortiz A. Diabetes mellitus tipo 2 en Perú: una revisión sistemática sobre la prevalencia e incidencia en población general. Rev perú med exp salud publica. 2019; 36:26-36. Doi: 10.17843/rpmesp.2019.361.4027

2. Armstrong EJ, Alam S, Henao S, et al. Multidisciplinary care for critical limb ischemia: current gaps and opportunities for improvement. J Endovasc Ther. 2019; 26(2):199-212. Doi: $10.1177 / 1526602819826593$

3. Conte MS, Bradbury AW, Kolh P, et al. Global vascular guidelines on the management of chronic limb-threatening ischemia. Eur J Vasc Endovasc Surg. 2019; 58(1S):S1-S109.e33. Doi: 10.1016/j. ejvs.2019.05.006
4. Almasri J, Adusumalli J, Asi N, et al. A systematic review and meta-analysis of revascularization outcomes of infrainguinal chronic limb-threatening ischemia. J Vasc Surg. 2019; 69(6S):126S-136S. Doi: 10.1016/j.jvs.2018.01.071

5. Mayor J, Chung J, Zhang Q, et al. Using the Society for Vascular Surgery Wound, Ischemia, and foot Infection classification to identify patients most likely to benefit from revascularization. J Vasc Surg. 2019; 70(3):776-85. e1. Doi: 10.1016/j.jvs.2018.11.039

6. Pereira C, Suh HP, Hong JPJ. Úlceras del pie diabético: importancia del manejo multidisciplinario y salvataje microquirúrgico de la extremidad. Rev Chil Cir. 2018; 70(6):535-43. Doi: 10.4067/s0718-4026201800060 0535

7. Vaquero C, Gutiérrez V, González-Fajardo J, et al. Procedimientos endovasculares. Valladolid: PROCIVAS; 2006.

8. Schmidt A, Bausback Y, Piorkowski M, et al. Retrograde recanalization technique for use after failed antegrade angioplasty in chronic femoral artery occlusions. J Endovasc Ther. 2012; 19(1):23-9. Doi: 10.1583/11-3645.1

9. Kawarada O, Yokoi Y. Retrograde 3-French popliteal approach in the supine position after failed antegrade angioplasty for chronic superficial femoral artery occlusion. J Endovasc Ther. 2010; 17(2):255-8. Doi: 10.1583/09-2966.1

10. Montero-Baker M, Schmidt A, Bräunlich S, et al. Retrograde approach for complex popliteal and tibioperoneal occlusions. J Endovasc Ther. 2008; 15(5):594-604. Doi: 10.1583/08-2440.1

Recibido: 20/04/2020

Aceptado: 25/06/2020 